\title{
Whole-body MR Imaging in Detecting Phosphaturic Mesenchymal Tumor (PMT) in Tumor-induced Hypophosphatemic Osteomalacia
}

\author{
Katsuyuki NaKanishi $^{1 *}$, Mio SAKaI ${ }^{1}$, Hisashi TAnaKa ${ }^{2}$, Hideki Tsubor $^{3}$, \\ Jun Hashimoto ${ }^{3}$, Nobuyuki Hashimoto ${ }^{4}$, and Noriyuki TomiYAmA ${ }^{2}$ \\ ${ }^{1}$ Department of Diagnostic Radiology, Osaka Medical Center for Cancer and Cardiovascular Diseases \\ 1-3-3, Nakamichi, Higashinari-ku, Osaka 537-8511, Japan \\ ${ }^{2}$ Department of Diagnostic Radiology, Osaka University Graduate School of Medicine \\ ${ }^{3}$ Department of Orthopedic Surgery, South-Osaka National Hospital \\ ${ }^{4}$ Department of Orthopedic Surgery, Osaka Medical Center for Cancer and Cardiovascular Diseases \\ (Received November 22, 2011; Accepted October 31, 2012; published online March 11, 2013)
}

We present 2 cases of tumor-induced osteomalacia (TIO). Both patients had histories of long-term bone and muscle pain. Laboratory data revealed hypophosphatemia. Wholebody magnetic resonance (MR) imaging (WB-MRI) clearly depicted a small subcutaneous mass in the left thigh of the first patient and a right acetabular mass in the second patient. These lesions were pathologically proven to be hemangiopericytoma-phosphaturic mesenchymal tumors (PMT).

\section{Keywords: phosphaturic mesenchymal tumor, tumor-induced osteomalacia, whole-body MRI}

\section{Introduction}

Tumor-induced osteomalacia (TIO) is a hypophosphatemic disease with renal phosphate wasting caused by paraneoplasm. Surgical removal of the responsible tumors is clinically essential. ${ }^{1}$ In 1987 , Weidner and Santa $\mathrm{Cruz}^{2}$ reported that most cases of TIO are histologically distinctive and unlike other mesenchymal tumors. Yoshioka's group ${ }^{3}$ coined the term "phosphaturic mesenchymal tumor mixed connective tissue variant" (PMTMCT) to describe this entity. Bone and muscle pain are generally the first clinical manifestations of PMTMCT and precede identification of the responsible tumor, which is often difficult because the tumors are predominantly small, slow-growing benign neoplasms. ${ }^{1,4}$ Recent evidence has shown that fibroblast growth factor 23 (FGF23) from the tumor causes TIO, which induces renal phosphate wasting. ${ }^{1}$ Skeletal survey by magnetic resonance (MR) imaging $^{5-7}$ is one radiologic tool for detecting PMTMCT. ${ }^{5-13}$ In the field of oncology, whole-body

\footnotetext{
*Corresponding author, Phone: +81-6-6972-1181, Fax: + 816-6981-3329, E-mail: je2k-nkns@asahi-net.or.jp
}

MR imaging (WB-MRI) is being improved and widely used for detecting metastatic bone tumors ${ }^{14,15}$ or lymph node metastases. ${ }^{16} \mathrm{We}$ describe our experience with 2 patients in whom PMTs were detected using WB-MRI.

\section{Case Reports}

Two patients diagnosed with TIO who presented with symptoms of long-term bone and muscle pain that suggested osteomalacia underwent WB-MRI. Scanning was performed using a 1.5-tesla wholebody scanner (Master Philips Medical System, Best, The Netherlands) and our previously reported institutional protocol for WB-MRI. ${ }^{14}$

Diffusion-weighted imaging (DWI) was performed using single-shot short $T_{1}$ inversion recovery-echo planar imaging (STIR-EPI) sequences with repetition time (TR), $6243 \mathrm{~ms}$, echo time (TE), $59 \mathrm{~ms}$, and inversion time (TI), $180 \mathrm{~ms}$, and bvalues of 0 and $600 \mathrm{~s} / \mathrm{mm}^{2}$. Five-mm slice thickness with one-mm overlap of the axial view was obtained during free breathing. Axial slices were obtained from the lower neck to the bottom of the pelvis using a 2-station approach. The field of view 
(FOV) of each station was $450 \mathrm{~mm}$, and the matrix was $112 \times 112$. The imaging time of each station was 5 min $25 \mathrm{~s}$. We then reconstructed the radial direction of maximal intensity projection (MIP) images of each station.

$\mathrm{T}_{1}$-weighted fast spin echo (SE) sequences included sagittal images of the total spine and coronal images of the whole body. We obtained 5 slice sections of $T_{1}$-weighted fast $\mathrm{SE}$ images $\left(\mathrm{T}_{1} \mathrm{WI}\right)$ (TR/ TE, 400/13 ms; echo train length [ETL], 4) of the total spine on the sagittal plane using a 3-station approach with slice thickness of $7 \mathrm{~mm}$. The FOV of each station was $300 \mathrm{~mm}$, and the matrix was $352 \times$ 264. Imaging time was $4 \min 33 \mathrm{~s}$.

Fast field echo (FFE) $\mathrm{T}_{1}$ WI (TR/TE, 100/4.6 ms; ETL, 128) of the whole body was performed on the coronal plane using a 6-station approach, with $300-\mathrm{mm}$ FOV of each station and a total of 32 whole-body coronal images of this sequence acquired from anterior to posterior with slice thickness of $7 \mathrm{~mm}$. The matrix was $240 \times 180$, and imaging time was 6 min $24 \mathrm{~s}$.

STIR sequences included sagittal images of the total spine and coronal images of the whole body. Five sections of STIR images (TR/TE/TI, 2500/ $70 / 170 \mathrm{~ms}$; ETL, 15) of the total spine were imaged on the sagittal plane using a 3-station approach with slice thickness of $7 \mathrm{~mm}, 300-\mathrm{mm}$ FOV of each station, and matrix, $288 \times 316$. Imaging time was 6 $\min 15 \mathrm{~s}$.

STIR images (TR/TE/TI, 1350/40/165 ms; ETL, 65) of the whole body were imaged on the coronal plane using a 6-station approach, with $300-\mathrm{mm}$ FOV of each station. A total of 32 wholebody coronal images were acquired from anterior to posterior with slice thickness of $7 \mathrm{~mm}$. The matrix was $320 \times 185$, and total imaging time was 6 $\min 24 \mathrm{~s}$.

The total examination time, including patient positioning, was within $50 \mathrm{~min}$.

\section{Case 1}

A 53-year-old woman complained of whole body pain, especially in both lower limbs, that began approximately 3 years' before. Laboratory findings included high serum alkaline phosphatase (ALP; $1432 \mathrm{U} / \mathrm{L}$ ), low inorganic phosphorus (IP; 1.6 $\mathrm{mg} / \mathrm{dl}$ ) and high fibroblast growth factor 23 (FGF $23 ; 172 \mathrm{pg} / \mathrm{mL}$ ). TIO was suspected, and WB-MRI revealed multiple fractures in the vertebral bodies. In addition, a small subcutaneous nodule that measured less than $20 \mathrm{~mm}$ in diameter was noted in her left thigh. The lesion was depicted as a hyperintense focus on both STIR and DWI but could not be detected on positron emission tomography using fluorodeoxyglucose (FDG-PET); it was considered the lesion responsible for the osteomalacia. The patient underwent surgery to remove the tumor, after which her serum phosphorus level recovered to the normal range and her symptoms improved. Hemangiopericytoma-phosphaturic mesenchymal tumor (PMT) was proven histologically (Fig. 1).

\section{Case 2}

A 40-year-old woman complained of right hip pain, which did not improve during the initial course of monitoring. A year after symptom onset, a tumor was detected in her right thyroid gland, and papillary carcinoma was proven in aspirated cytology. At the same time, CT detected a tumor in the right ilium that was suspected to be a metastatic bone tumor from the thyroid carcinoma. Despite the bone lesion, her serum thyroglobulin level recovered immediately after total thyroidectomy.

Moreover, low serum IP $(1.4 \mathrm{mg} / \mathrm{dl})$ continued and high FGF $23(135 \mathrm{pg} / \mathrm{mL})$ was proven. Therefore, we suspected the bone lesion was not metastatic, performed open biopsy, which proved hemangiopericytoma-PMT, and then performed WBMRI (Fig. 2). Because the tumor was considered too large to remove surgically, transcatheter arterial embolization (TAE) was performed, and tumor size decreased and serum IP recovered temporarily to the normal range.

\section{Discussion}

TIO is a paraneoplastic disorder with hypophosphatemia, phosphaturia, inappropriately low serum levels of 1,25-dihydroxyvitamin D for hypophosphatemia, skeletal undermineralization. Its clinical symptoms, such as pathologic bone fracture, severe bone pain, and regular muscle weakness, resemble those of X-linked hypophosphatemic rickets (XLH). Tumors responsible for TIO are usually benign rather than invasive. The important clinical issues for the patient are generalized, debilitating osteomalacia and rickets. ${ }^{1,4}$ Hypophosphatemia in TIO results from an excessive renal loss of phosphate, with a low ratio of phosphorus tubule maximum (TmP) to glomerular filtration rate (GFR) and a low percentage of tubular reabsorption of phosphate (TRP). Evidence shows that patients who undergo tumor removal-the primary treatment for TIO-enjoy complete relief from debilitating symptoms. ${ }^{1,17}$

Various modalities, such as CT, MR imaging, ${ }^{5-7}$ FDG-PET, ${ }^{9-11}{ }^{111}$ In-pentetreotide, octreotide scin- 

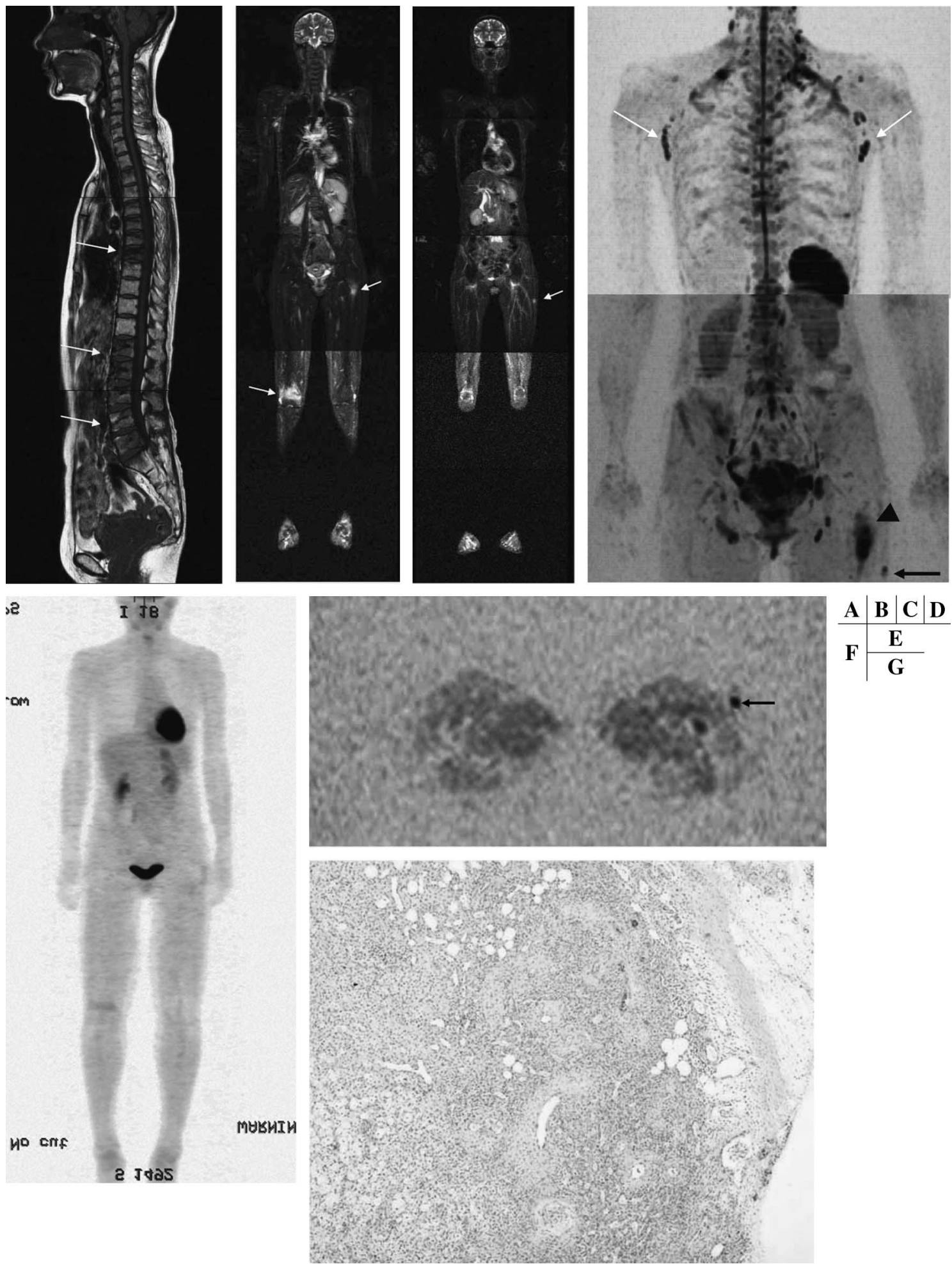

Fig. 1. A 53-year-old woman (Case 1). (A) Total spine $T_{1}$-weighted image shows multiple linear low intensity in most parts of the lumbar vertebral bodies (white arrows). (B) Whole body STIR coronal image shows high intensity in the knee and femur that reflects bone marrow edema due to fracture (white arrows). (C) Whole-body STIR coronal image ventral from Fig. 1B shows a small area of high intensity in the left femoral subcutaneous region (white arrow). (D) A maximum intensity projection (MIP) image of diffusion-weighted imaging (DWI) shows this lesion as a strong signal as Fig. 1C (arrow). There is also strong signal in the left femur that corresponds to a fracture (arrowhead). In both axillae, nodular areas of strong signal are considered to be nonspecific lymph nodes (white arrows). (E) Original axial DWI shows small high intensity in the left femoral subcutaneous region (arrow). (F) Wholebody positron emission tomography using fluorodeoxyglucose (FDG-PET) image does not depict this lesion. (G) Histologically, hemangiopericytoma-phosphaturic mesenchymal tumor (PMT) was proved. 

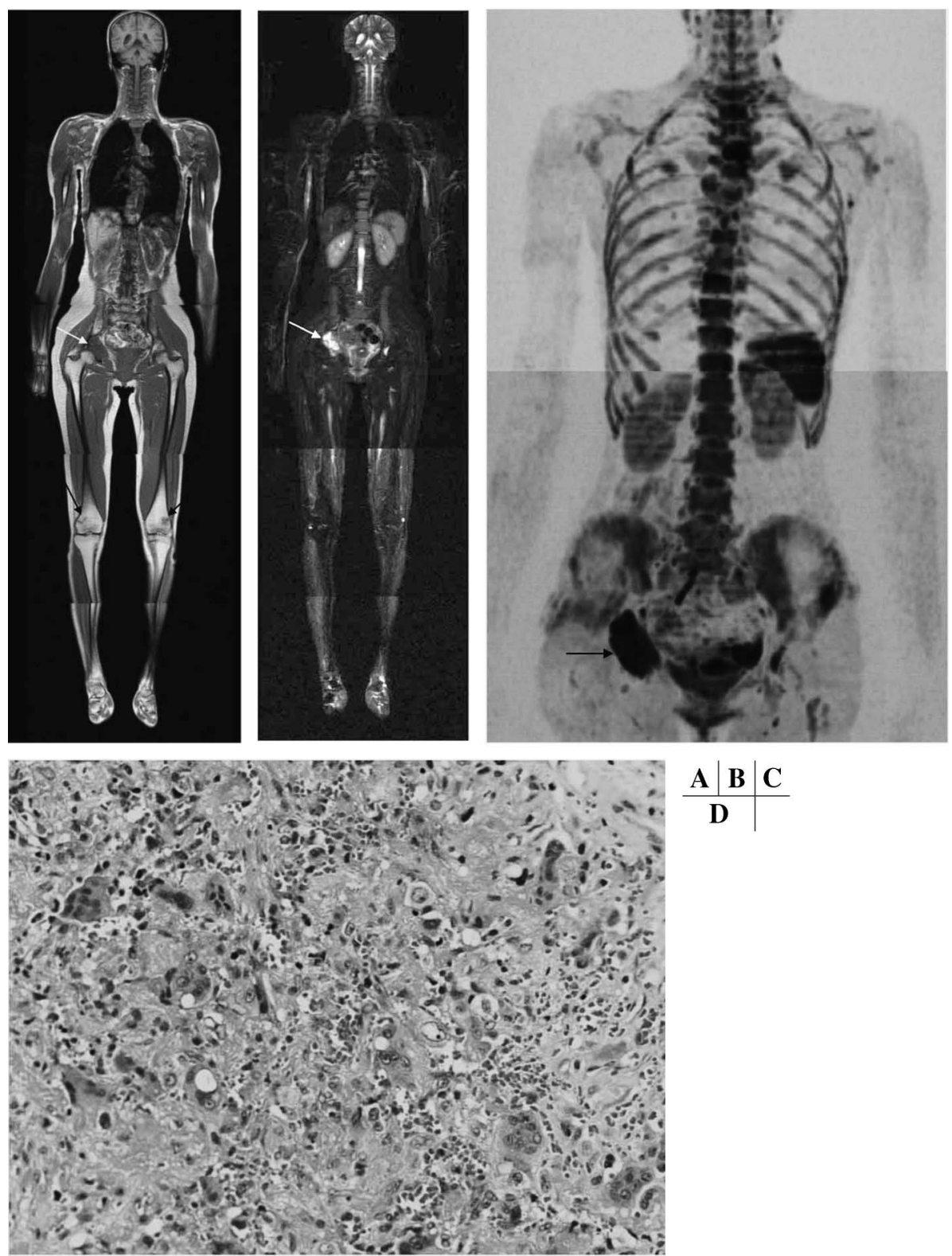

\begin{tabular}{c|c|c}
$\mathbf{A}$ & $\mathbf{B}$ & $\mathbf{C}$ \\
\hline $\mathbf{D}$ &
\end{tabular}

Fig. 2. A 40-year-old woman (Case 2). (A) $T_{1}$-weighted coronal image shows a mass with low intensity in the right acetabulum (white arrow), which was proved a phosphaturic mesenchymal tumor (PMT) by open biopsy. Linear areas of low intensity in the bilateral knee joints correspond to old fractures (arrows). (B) STIR coronal image also shows high intensity in the right acetabulum (white arrow). (C) A maximum intensity projection (MIP) image of diffusion-weighted imaging (DWI) also shows high intensity in the right acetabulum (arrow). (D) Histology proved hemangiopericytoma-phosphaturic mesenchymal tumor (PMT) was proved.

tigraphy ${ }^{12,13}$ and systemic venous sampling ${ }^{1,17,18}$ have been used to localize the tumor of TIO. In what we believe is the largest report from a single center, Jagtap and associates analyzed 9 patients with TIO, ${ }^{11}$ using ${ }^{18} \mathrm{~F}$-FDG PET to localize the tumors. Several other studies report using FDG PET to localize the tumor of TIO, most used MR imaging then to verify FDG-PET findings rather than as a screening tool. We found no reports of tumors with TIO depicted by WB-MRI rather than FDG-PET, as in our Case 1. Previous reports describe the tumors causing TIO as sometimes small and predominantly slow-growing neoplasms of benign mesenchymal origin. A large mass, such as our Case 2, might be rare. Considering the characteristics of this tumor, it might not be seen as in- 
creased uptake of FDG-PET utilization.

In contrast, MR imaging has no radiological exposure and offers excellent contrast resolution of bone, soft tissue, and subcutaneous regions. We believe 3 publications have reported use of MR imaging for skeletal survey to detect the lesion of TIO. ${ }^{5-7}$

Avia's group ${ }^{5}$ used a $0.5 \mathrm{~T}$ unit to perform an MR skeletal survey that included axial STIR images of the cranium, thorax, and abdomen as well as coronal images of the proximal appendicular skeleton. Fukumoto and colleagues ${ }^{6}$ described the division of major bones in the body into several groups and performed screening using $\mathrm{T}_{1} \mathrm{WI}$ and $\mathrm{T}_{2} \mathrm{WI}$. Dissanayake and associates ${ }^{7}$ described acquisition of STIR images using a $48-\mathrm{cm}$ FOV in the coronal and axial planes at the neck, thorax, abdomen, pelvis, and legs. However, these reports did not detail the circumstances for their methodology, such as whether examinations were performed in one sitting or installments, nor did they state total examination times. We applied the imaging protocol we generally use as to screen for metastatic bone tumor ${ }^{14}$ to detect TIO. MR imaging skeletal survey was performed in one sitting. Total examination time, including patient positioning, was within $50 \mathrm{~min}$.

We used coronal STIR images from the cranium to the leg and sagittal STIR images of the total spine. These sequences resembled those described in previous publications. ${ }^{14}$ In addition, we employed axial DWI from the lower neck to the bottom of the pelvis, which was not previously reported. The tumors of our 2 cases were well depicted in both STIR and DWI, findings considered nonspecific. However, the few experiences reported have not proved the utility of DWI for detecting TIO. Various malignancies appear hyperintense on DWI because of their high cellularity. ${ }^{14}$ However, both our cases of hemangiopericytoma-PMT were slowgrowing benign neoplasms, and their high signal on DWI may have derived from $T_{2}$ shine-through effect. Moreover, DWI has less spatial resolution than STIR, so high resolution STIR images should probably be prioritized to detect PMT. The value of DWI for detecting PMT is controversial and requires further investigation.

We used WB-MRI to analyze cases of TIO, which facilitates detection of fracture sites and their condition. ${ }^{14-16}$ Coronal views of $\mathrm{T}_{1} \mathrm{WI}$ and STIR of WB-MRI are useful for detecting fractures of the appendicular skeleton, and sagittal views of conventional $\mathrm{T}_{1} \mathrm{WI}$ and STIR of total spine images are useful for detecting fractures of vertebral bodies.

In conclusion, WB-MRI is a useful noninvasive procedure to detect PMT. STIR should be used preferentially for detecting PMT.

\section{References}

1. Nasu T, Kurisu S, Matsuno S, et al. Tumor-induced hypophosphatemic osteomalacia diagnosed by the combinatory procedures of magnetic resonance imaging and venous sampling for FGF23. Intern Med 2008; 47:957-961.

2. Weidner N, Santa Cruz D. Phosphaturic mesenchymal tumors. A polymorphous group causing osteomalacia or rickets. Cancer 1987; 59: 14421454.

3. Yoshioka K, Nagata R, Ueda M, et al. Phosphaturic mesenchymal tumor with symptoms related to osteomalacia that appeared one year after tumorectomy. Intern Med 2006; 45:1157-1160.

4. Folpe AL, Fanburg-Smith JC, Billings SD, et al. Most osteomalacia-associated mesenchymal tumors are a single histopathologic entity: an analysis of 32 cases and a comprehensive review of the literature. Am J Surg Pathol 2004; 28:1-30.

5. Avila NA, Skarulis M, Rubino DM, Doppman JL. Oncogenic osteomalacia: lesion detection by MR skeletal survey. AJR Am J Roentgenol 1996; 167 : 343-345.

6. Fukumoto S, Takeuchi Y, Nagano A, Fujita T. Diagnostic utility of magnetic resonance imaging skeletal survey in a patient with oncogenic osteomalacia. Bone 1999; 25:375-377.

7. Dissanayake AM, Wilson JL, Holdaway IM, Reid IR. Oncogenic osteomalacia: culprit tumour detection whole body magnetic resonance imaging. Intern Med J 2003; 33:615-616.

8. Lee HK, Sung WW, Solodnik P, Shimshi M. Bone scan in tumor-induced osteomalacia. J Nucl Med $1995 ; 36: 247-249$.

9. Dupond JL, Mahammedi H, Magy N, Blagosklonov O, Meaux-Ruault N, Kantelip B. Detection of a mesenchymal tumor responsible for hypophosphatemic osteomalacia using FDG-PET. Eur J Intern Med 2005; 16:445-446.

10. Khadgawat R, Singh Y, Kansara S, et al. PET/CT localisation of a scapular haemangiopericytoma with tumour-induced osteomalacia. Singapore Med J 2009; 50:e55-e57.

11. Jagtap VS, Sarathi V, Lila AR, et al. Tumor-induced osteomalacia: A single center experience. Endocr Pract 2010; 17:177-184.

12. Seufert J, Ebert K, Müller J, et al. Octreotide therapy for tumor-induced osteomalacia. $\mathrm{N}$ Engl $\mathrm{J}$ Med 2001; 345:1883-1888.

13. Duet M, Kerkeni S, Sfar R, Bazille C, Lioté F, Orcel $\mathrm{P}$. Clinical impact of somatostatin receptor scintigraphy in the management of tumor-induced osteomalacia. Clin Nucl Med 2008; 33:752-756.

14. Nakanishi K, Kobayashi M, Nakaguchi K, et al. Whole-body MRI for detecting metastatic bone 
tumor: diagnostic value of diffusion-weighted images. Magn Reson Med Sci 2007; 6:147-155.

15. Gutzeit A, Doert A, Froehlich JM, et al. Comparison of diffusion-weighted whole body MRI and skeletal scintigraphy for the detection of bone metastases in patients with prostate or breast carcinoma. Skeletal Radiol 2010; 39:333-343.

16. Schmidt GP, Reiser MF, Baur-Melnyk A. Wholebody MRI for the staging and follow-up of patients with metastasis. Eur J Radiol 2009; 70:393400 .
17. Takeuchi Y, Suzuki H, Ogura S, et al. Venous sampling for fibroblast growth factor-23 confirms preoperative diagnosis of tumor-induced osteomalacia. J Clin Endocrinol Metab 2004; 89:39793982.

18. van Boekel G, Ruinemans-Koerts J, Joosten F, Dijkhuizen P, van Sorge A, de Boer H. Tumor producing fibroblast growth factor 23 localized by two-staged venous sampling. Eur J Endocrinol 2008; 158:431-437. 